Kevin McGuire

November 1984 
CONTENTS

1. 0

2. 0

3. 0

4. 0

5. 0

5. 1

5. 1.1

5. 1.2

5. 1.3

5. 1.4

5. 1.5

5. 1.6

5. 1.7

5. 1.8

5. 1.9

5. 1.10

5. 1.11

5. 1. 12

5. 2

5. 2. 1

5. 2. 2

5. 2. 3

5. 2.4

5. 3

5. 3. 1

5. 3.2

5. 3.3

5. 3.4

5. 3.5

5. 3.6

5. 3. 7

5. 3. 8

5. 3.9

5. 3. 10

5. 3. 11

5. 3.12
Abstract

GPIB Routine Overview

System Qverview

CAMAC Interface

GPIB Routines

Primitive routines

GPATN

GPDATN

GPDREN

GPDUAI

GPIFC

GPREAD

GPREADCH

GPREN

GPSTATUS

GPUAI

GPWR ITE

GPWR I TECH

Universal and Addressed Commands.

GPGET

GPLLD

GPSPD

GPSPE

Miscellaneous Routines.

GPSPSTAT

GPSRO

GPPOLL . . 22

GPRDAT . . . . . . . . . . . . . . . . . . . . 23

GPRDATSTR . . . . . . . . . . . . . . . . . . . 24

GPTRIG... . . . . . . . . . . . . . . . . . . . 25

GPULISTEN . . . . . . . . . . . . . . . . . . . . 26

GPUNL • . . . . . . . . . . . . . . . . . . 27

GPUNT . . . . . . . . . . . . . . . . . . . . . . 27

GPUTALK . . . . . . . . . . . . . . . . . . . 28

GPXMIT . . . . . . . . . . . . . . . . . . . . . . 29

GPXMITSTR . . . . . . . . . . . . . . . . . . . . . 30 


\section{0 Abstract}

This package has been developed for communicating with devices on the GPIB (General Purpose Interface Bus - IEEE Std. 488). Programs and subroutines which call the routines in this package communucate with devices on the GPIB via a GPIB controller residing in a CAMAC crate. CAMAC access is done in 16-bit transfers. The target hardware is a DEC VAX 730 which communicates with a byte-serial CAMAC loop through a Jorway Model 411 PDP-11 CAMAC Interface. CAMAC in turn, communicates with the GPIB through a Kinetic Systems Model 3388-G1A GPIB Interface. Although this is the hardware that is used at our installation, the software is designed to be used on other systems with a minimum of modifications. 
2. O GPIB Routine Dverview

Primitives

GPATN

GPDATN

GP IFC

GPREN

GPDREN

GPREAD

GPREADCH

GPWR ITE

GPWR I TECH

GPSTATUS

GPUAI

GPDUAI
Mnemonic/Function

Enables AtTeNtion

Disables AtTeNtion

Performs an "Interface Clear"

Remote ENable

Disable Remote ENable

READs the Input Register of the controller as a binary integer

READs the Input Register of the controller as a CHaracter

WRITEs a binary integer to the

Output Register of the controller

WRITEs a CHaracter to the Dutput Register of the controller

Reads the controller's Status Register

Enables the Universal and Audressed Command

Inhibit - This command is used when the

Kinetic Systems Model 3388 GPIB Interface

is being used as the system controller. I

believe this command is unique to this

particular controlier).

It affects the haridshake.

Disables the Universal and Addressed Command Inhibit - Use this when the 3388 is not the system controller. 
GPIB Routine Overview (cont'd.):

Universal and Addressed Commands

GPLLD

GPGET

GPSPE

GPSPD

General-Purpose Routines

GPUNT

GPUNL

GPUTALK

GPULISTEN

GPXMIT

GPXMITSTR

GPRDAT

GPRDATSTR

GPSPSTAT

GPPOLL

GPTRIG

GPSRQ
Mnemonic/Function

Local Lock-Out

Group Execute Trigger

Serial Poll Enable

Serial Poll Disable

Function

Untalk

Unlisten

Sets a talker

Sets a listener

Transmits an array

Transmits a character string

Reads an array

Reads a character string

Reads Serial Poll Status Words

Polls devices

Selectively triggers Waits for a Service Request 


\section{O System Qverview}

The following section examines details which are unique to the system under which this package was developed. While it describes the way we have implemented these routines, it is not the only way that the routines in this package may be used. An attempt has been made to make this package flexible and general enough so that transporting it to other systems can be done with a minimum number (if any) of modifications.

The hardware that we use on our system consists of a VAX 730 computer which communucates with the CAMAC crates through a Jorway Model 411 PDP-11 CAMAC Interface. We use a special driver which handles the "Jorway"s as any other I/0 device. The software interface between the FORTRAN routines in this package and CAMAC is provided by ANSI/IEEE std758-1979 standard CAMAC routines. All of the GPIB routines transfer data to/from CAMAC using 16-bit transfers. This makes the package compatible with computers which have data busses less than 24 bits wide (most noteable PDP-11s) and it also provides increased speed and efficiency (over 24-bit transfers).

This package was designed for use at the Fermilab Development and Test Facility for use in programs which are used to test Tevatron I magnets. The overall scheme for testing these magnets requires that all hardware used in measurements will be entered into data bases. Information stored in these data bases will include the readable IDs of the particular piece of hardware, the FNAL IDs (an ID given to it by the laboratory for purposes of inventory), the calibration coefficients, and the Branch, Crate, slot Number, subAddress, and Function code for all CAMAC hardware. All records in these databases are date and time-stamped so that a corelation can be made between data collected and which hardware was used to collect the data.

All measurements go through an initialization phase where the data bases are queried (using a Digital Equipment Corporation database query package known as DATATRIEVE) to determine which pieces of hardware should be used for the measurement and then the hardware itself is queried for its IDs. If the IDs do not match, the mis-match is flagged and the measurement will not proceed until either the proper piece of hardware is used cor the reason that it was read improperly is solved) or the data bases are repaired. (Sometimes entries in the data bases are made incorrectly.) 
All of the measurement programs are written in FORTRAN. As the databases are queried through FORTRAN routines which interface with DATATRIEVE, COMMON blocks are filled which contain the information necessary to do things with the hardware.

The relevance which the preceeding discussion has with the GPIB package is that the CAMAC Branch, Crate, slot Number, subAddress, and Function codes to be used in controlling the GPIB bus are all entered into a data base known as CAMAC_OPERATION.

The flexibility that this offers is that any GPIB controller may be used through CAMAC without the need to re-write all of the GPIB code. The GPIB controller we are using is the Kinetic Systems Model 3388-G1A GPIB Interface Module. If a different GPIB controller module were to be installed in its place, only the data base called CAMAC_DPERATION would need to be changed. No editing and re-compiling would need to be done to the source code.

For those who do not have DATATRIEVE or have it, and don't wish to use it for the same purposes which we use it, a block of DATA statements is provided which has all of the necessary information hard-coded. If your GPIB controller uses subaddresses (for different GPIB functions) that are different from the controller that we use, you must consult the manual for your controller and edit the file containing the DATA statements to make the necessary corrections. Those operations which run under VAX/UMS FORTRAN 77 may use the INCLUDE statement to easily insert these parameters into coMMON blocks at compile-time. otherwise you will have to edit these into the module which declares the COMMON block. 


\section{O CAMAC Interface}

Since the GPIB package is so intimately tied to CAMAC, the Fermilab Magnet Development and Test Facility CAMAC Package is distributed along with it. This package adheres to IEEE Std. 758-1979 FORTRAN calling conventions.

For those installations without DATATRIEVE:

Since the purpose of DATATRIEVE at our installation is basically to fill COMMON blocks with values obtained in out databases, the use of DATATRIEVE is not central to the application of this GPIB package as long as the COMMON blocks are filled somehow. To this end, a subroutine (GPIBFILL) has been provided to fill these COMMON blocks. In order to fill these COMMON blocks, GPIBFILL has an INCLUDE statement which INCLUDEs a text module called GPIBPARAM. If you are using the same GPIB controller as we are (the Kinetic Systems Model 338B-G1A GPIB Interface Controller), you may use this text module (GPIBPARAM) without any changes. If you are using a different GPIB controller, you may need to edit GPIBPARAM to reflect different function codes and subaddresses required to perform different GPIB interface functions. Needless to say lout easy to overlook), your program must call GPIBFILL before trying to make any GPIB calls.

For those installations wishing to use DATATRIEVE:

If you wish to use DATATRIEVE, you have two choices. You may set up your own data base and write your own routines to fill the COMMON block HDW_GPIB, or you may duplicate out system exactly and use our FORTRAN DATATRIEVE access routines. 


\section{O GPIB Routines}

\section{1 Primitive routines}

The primitive routines make the interface between CAMAC and the GPIB controlier. They exist for the sole purpose of doing CAMAC accesses. Therefore, the user can call GPIB routines without even thinking about CAMAC. All GPIB routines can be built from these primitives. In fact, all of the "non-primitive" routines (including the Universal and Addressed commands) are built from these primitives. There are also two "elaborated" primitive routines. These are GPREADCH and GPWRITECH. These do the same function as GPREAD and GPWRITE respectively, with the exception that the data is transferred as an ASCII character, rather than as an integer.

\section{1. 1 GPATN}

Function:

Sets the GPIB bus in ATTENTION mode."

\section{Calling Sequence:}

CALL GPATN \& )

Input/Output:

Inputs (from the COMMON block HDW_GPIB) :

$\begin{array}{ll}\text { GPIB_ATN_ADDR } & \text { INTEGER } * 4 \\ \text { GPIB_ATN_FUNC } & \text { INTEGER } * 4 \\ \text { Q_TRY } & \text { INTEGER } * 4\end{array}$

CAMAC address - includes Software Channel, Branch, Crate, and subAddress CAMAC Function code to Enable Attention

The number of times to loop for a a responsa 


\section{1.2 GPDATN}

Function:

Disables ATteNtion on the GPIB bus.

Calling Sequence:

GPIBSTAT = GPDATN ( )

Input/output:

Inputs (from the COMMON block HDW_GPIB) :

GPIB_DATN_ADDR INTEGER*4 CAMAC address - inciudes

Software Channel, Branch,

GPIB_DATN_FUNC INTEGER*4

Q_TRY INTEGER *4

Crate, and subAddress

CAMAC Function code to

Disable Attention

The number of times to loop

for a $Q$ response

5. 1. 3 GPDREN

Function:

Disables the Remote ENable on the GPIB bus.

Calling Sequence:

GPIBSTAT = GPDREN ( )

Input/Output :

Inputs (from the COMMON block HDW_GPIB) :

GPIB_DREN_ADDR INTEGER*4 CAMAC address - includes

Software Channel, Branch,

GPIB_DREN_FUNC INTEGER*4

Crate, and subAddress

Q_TRY INTEGER*4

CAMAC Function code to

Disable Remote ENable

The number of times to $100 p$

for a $Q$ response 
5. 1. 4 GPDUAI

Function:

Disables the Universal and Addressed Command Inhibit.

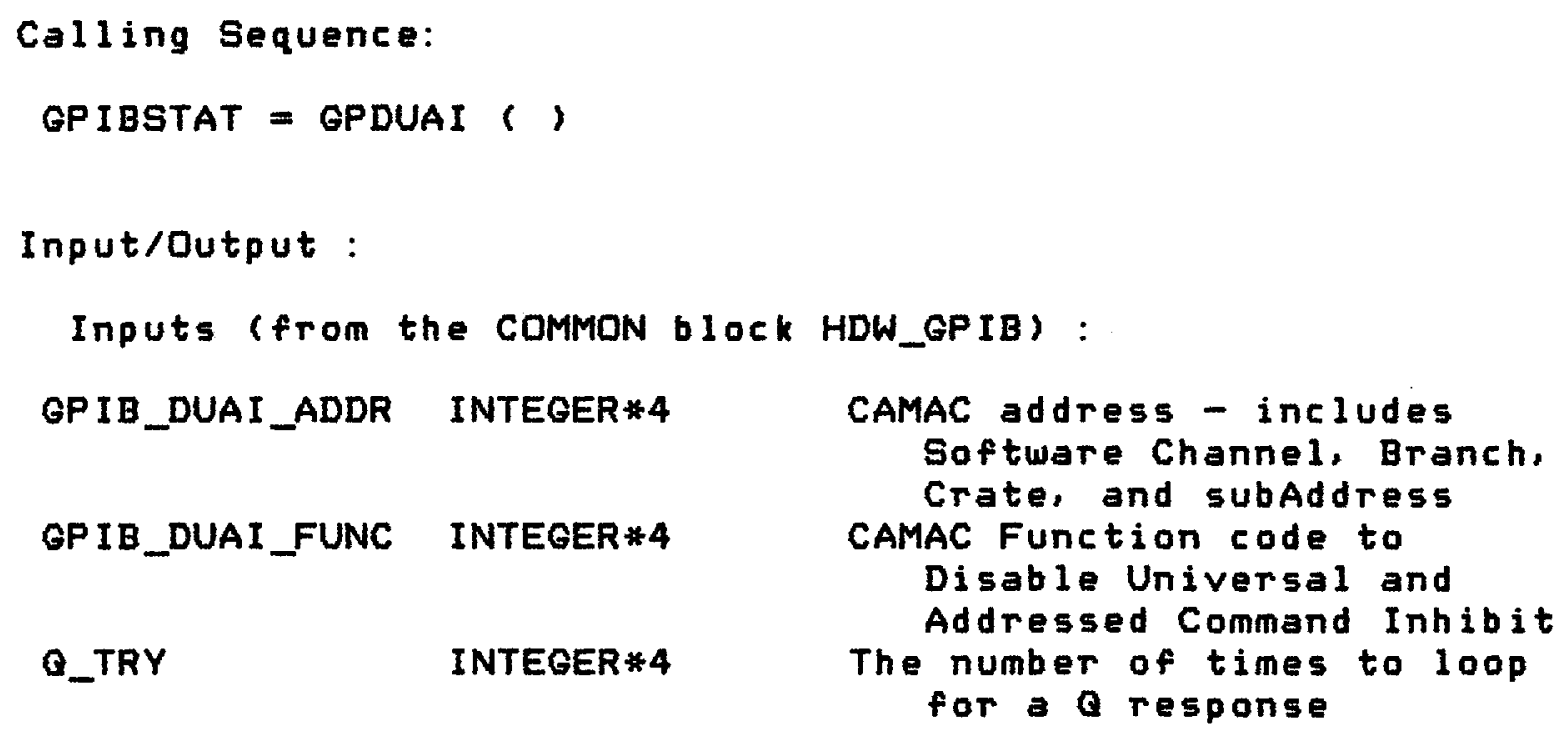

5. 1. 5 GPIFC

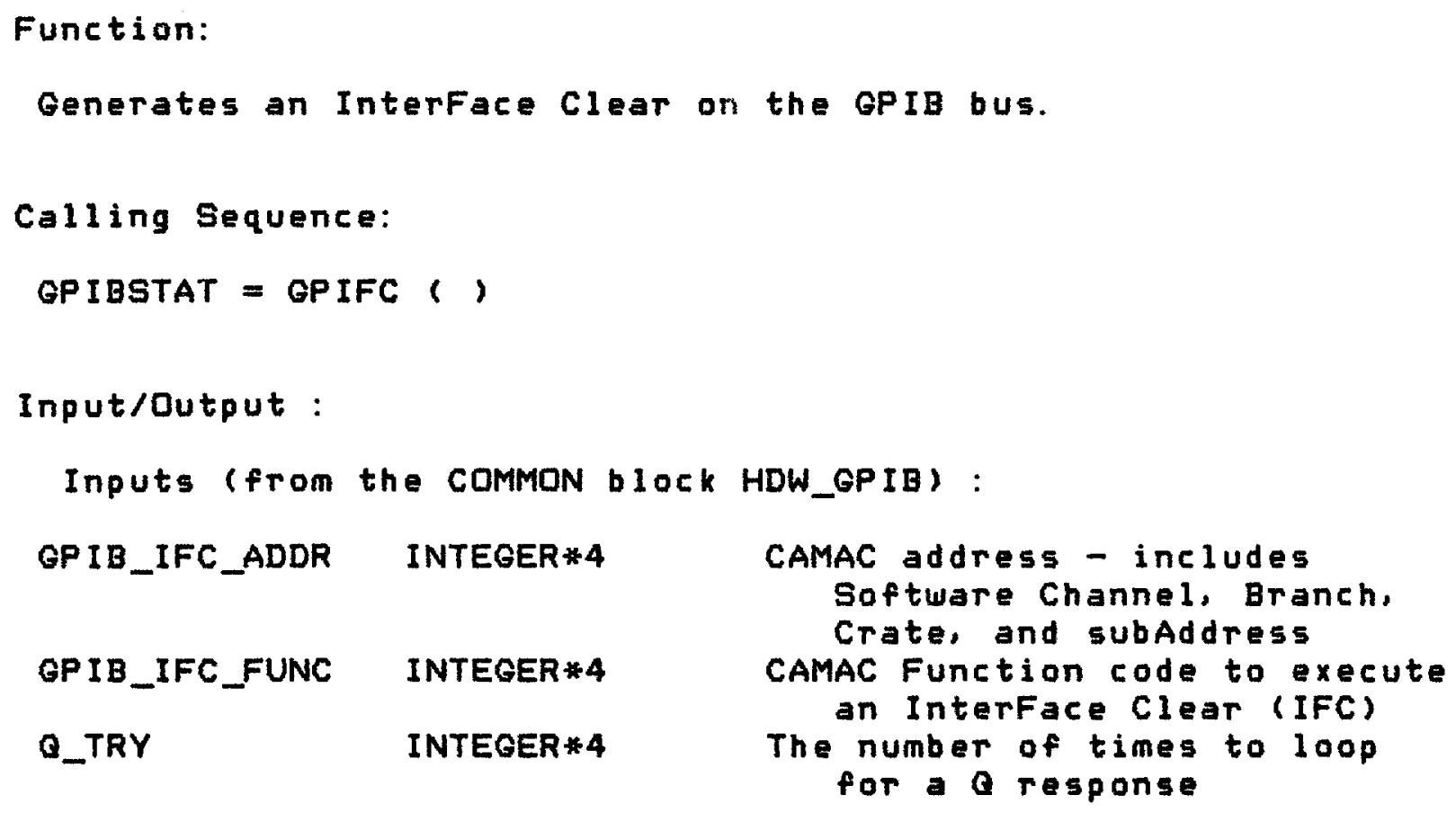




\section{1.6 GPREAD}

Function:

READs one data word (zero-extended byte) from the GPIB bus.

Calling Sequence:

GPIBSTAT = GPREAD (READ_WORD)

Input/output:

Inputs (from the COMMON block HDW_GPIB):

GPIB_READ_IN_ADDR

GPIB_READ_IN_FUNC

Q_TRY

Outputs:

READ_WORD
INTEGER *4

INTEGER *4

INTEGER*4

INTEGER*2
CAMAC address includes Software Channel, Branch, Crate, and subAddress CAMAC Function code to Read the Input Register of the controller

The number of times to loop for a $Q$ response

A zero-extended byte of data from GPIB 


\section{1. 7 GPREADCH}

Function:

READs one ASCII character from the GPIB bus.

Calling Sequence:

GPIBSTAT = GPREADCH ( ASCII_CHARACTER )

Input/Output:

Inputs (from the COMMON block HDW_GPIB):

GPIB_READ_IN_ADDR

GPIB_READ_IN_FUNC

Q_TRY

Qutputs:

ASCII_CHARACTER
INTEGER *4

INTEGER * 4

INTEGER *4

CHARACTER * 1
CAMAC address includes Software Channel, Branch, Crate, and subAddress CAMAC Function code to Read the Input Register of the controller

The number of times to loop for a a response

An ASCII character from GPIB 
5. 1. B GPREN

Function:

Sets GPIB devices in REMOTE, where operating parameters may be set by sofware, rather than manualiy.

Calling Sequence:

GPIESTAT = GPREN ( )

Input/Output:

Inputs (from the COMMON block HDW_GPIB):

GPIB_REN_ADDR INTEGER*4

GPIB_REN_FUNC INTEGER*4

Q_TRY INTEGER*4
CAMAC address - includes Softuare Channel. Branch, Crate, and subAddress CAMAC Function code to Enable Remote

The number of times to loop for a $Q$ response 
5. 1.9 GPSTATUS

Function:

Reads the status register in the GPIB Interface Module.

Calling Sequence:

GPIBSTAT = GPSTATUS ( STATUS_WDRD )

Input/Qutput:

Inputs (from the COMMON block HDW_GPIB) :

GPIB_READ_STAT_ADDR INTEGER*4 CAMAC address -

includes Software

Channel, Branch,

GPIB_READ_STAT_FUNC INTEGER*4

Crate, and subAddress

CAMAC Function code to

Read the Status

Register of the

Q_TRY

INTEGER *4

contraller

The number of times to loop for a $Q$ response

Dutputs:

STATUS_WORD

INTEGER *2

The status byte from the controller 
5. 1. 10 GPUAI

Function:

Enables the Universal and Addressed Command Inhibit.

\section{Calling Sequence:}

GPIBSTAT = GPUAI ( )

Input/Qutput:

Inputs (from the COMMON block HDW_GPIB):

GPIB_UAI_ADDR INTEGER*4

GPIB_UAI_FUNC INTEGER*4

CAMAC address - includes Software Channel, Branch, Crate, and subAddress CAMAC Function code to Enable the Universal and

o_tRY

INTEGER *4 Addressed command Inhibit The number of times to loop for a O response 


\section{1. 11 GPWR ITE}

Function:

WRITEs a byte to the GPIB bus.

Calling Sequence:

GPIBSTAT = GPWRITE ( WRITE_WORD )

Input/Output:

Inputs (from the COMMON block HDW_GPIB):

GPIB_WRITE_OUT_ADDR INTEGER*4

GPIB_WRITE_DUT_FUNC

INTEGER *4

O_tRY

INTEGER *4

Input (from argument 1 ist):

WR ITE_WORD

\author{
INTEGER*2
}

CAMAC address includes Software Channel, Branch. Crate, and subAddress CAMAC Function code to write to the Qutput Register of the controller

The number of times to loop for a $Q$ response
A word of data to be written to GPIB 
5. 1. 12 GPWRITECH

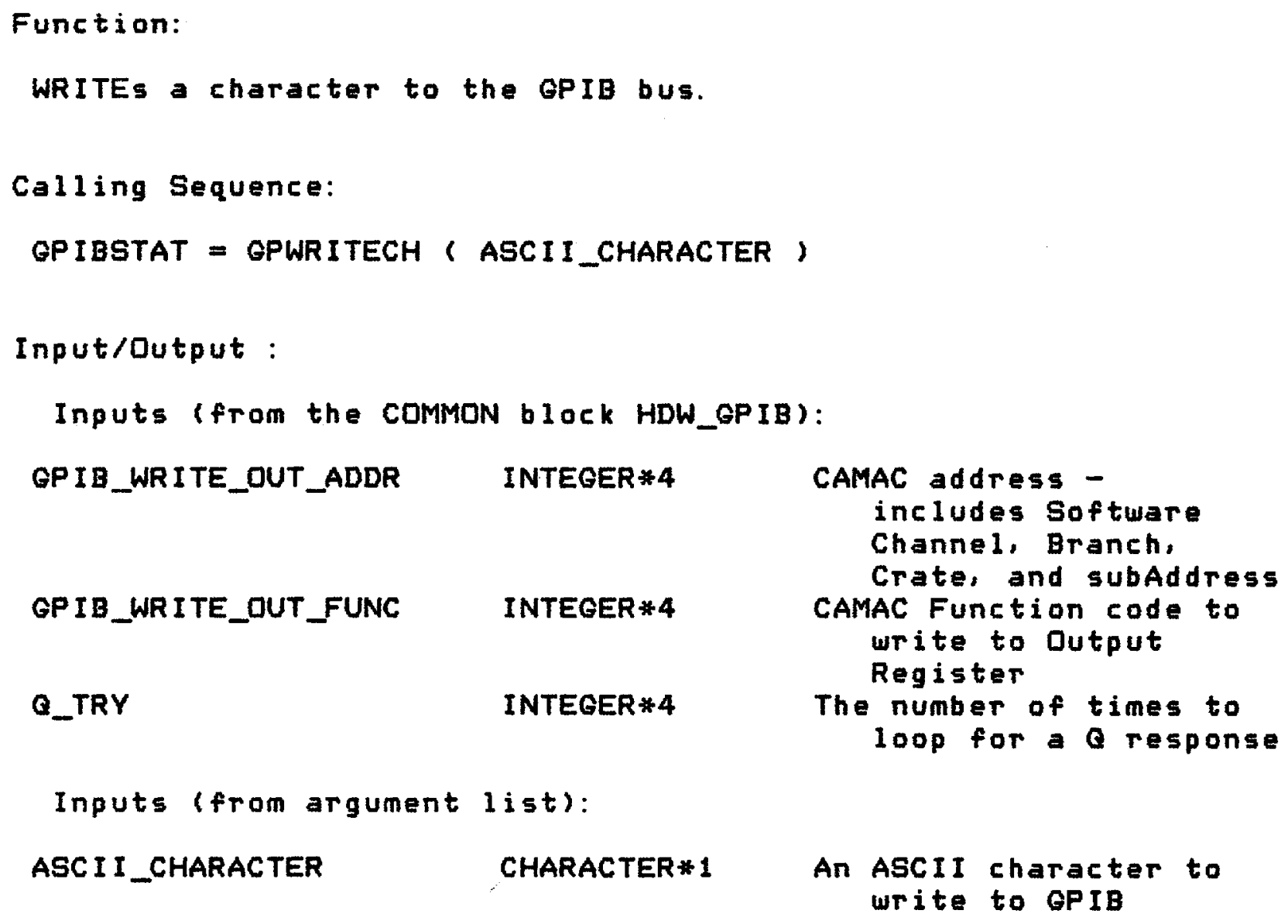


5. 2 Universal and Addressed Commands

These routines perform the GPIB Universal and Addressed Commands. Not all Universal and Addressed Commands have been implemented in this package, since there was never a need for them at our installation.

5. 2. 1 GPGET

Function:

This routine performs a Group Execute Trigger on the GPIB bus.

Calling Sequence:

GPIBSTAT = QPGET ( )

Input/Output:

Inputs (from the COMMON block HDW_GPIB):

GPIB_TALK_ADDR

INTEGER *2

Talk address of the controller

Dutputs:

none

5. 2.2 GPLLD

Function:

This routine performs the GPIB Universal Command 'Local Lockout (LLO) on the GPIB (IEEE Std 48B) bus.

Calling Sequence:

GPIBSTAT = GPLLD ( )

Input/Output:

none 
Page 20

5. 2.3 GPSPD

Function:

This routine performs a Serial Poll Disable on the GPIB bus.

Calling Sequence:

GPIBSTAT = GPSPD ( )

Input/Output:

none

5.2. 4 GPSPE

Function:

This routine performs a Serial Poll Enable on the GPIB bus.

Calling Sequence:

GPIBSTAT = GASPE ( )

Input/Output:

none 


\section{3 Miscellaneous Routines}

5. 3. 1 GPSPSTAT

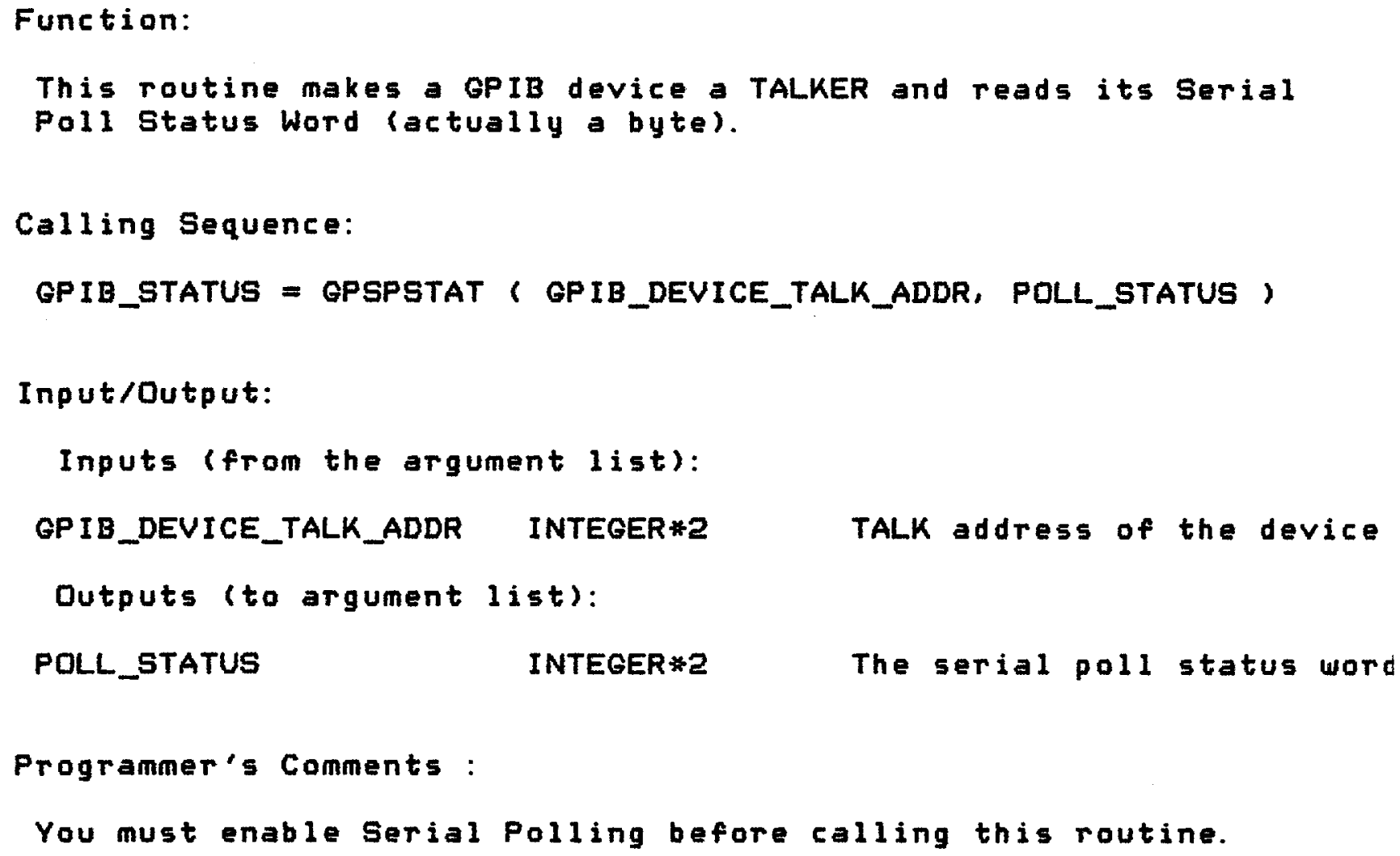

5. 3. 2 GPSRQ

Function:

This routine reads the status register in the GPIB controller and returns to its caller when the SRQ bit is set.

Calling Sequence:

STATUS = GPSRQ ( )

Input/output:

none 


\section{3. 3 GPPOLL}

Function:

This routine performs a serial poll on the specified devices and returns an array of their serial poll status words.

Calling Sequence:

GPIB_STATUS = GPPOLL ( POLL_TALK_ADDR, POLL_COUNT, POLL_STATUS )

Input/Output:

Inputs (from the COMMON block HDW_GPIB):

GPIB_LISTEN_ADDR

INTEGER*2

GPIB_READ_STAT_MLA

INTEGER *2

Q_TRY

INTEGER* 4

INTEGER *4

Inputs (from the argument 1 ist):

POLL_TALK_ADDR (POLL_CDUNT) INTEGER*?

POLL_COUNT

Dutputs (to argument list):

POLL_STATUS (POLL_COUNT) INTEGER*2
LISTEN address of interface module

The bit in the interface module which is set when the module is a 1 istener

The number of times for CAMAC to attempt an operation before giving up.

An array containing the TALK addresses of devices to be polled The number of devices to $p 011$

An array of serial poll status words 


\section{3. 4 GPRDAT}

Function:

Reads a word array containing ASCII data from the GPIB bus.

\section{Calling Sequence:}

GPIB_STATUS = GPRDAT ( GPIB_DEVICE_ADDR, DEVICE_COUNT, 2 DATA_ARRAY, BYTES_TRANSMITTED ,

Input/Dutput:

Input:

GPIB_DEVICE_ADDR

DEVICE_COUNT

Qutput:

DATA_ARRAY

BYTES_TRANSMITTED
INTEGER*2 (DEVICE_COUNT)

Array of GPIB addresses

INTEGER*4

The sum of the number of TALKERS and LISTENERS on the GPIB bUS.

\section{INTEGER *2 (BYTES TRANSMITTED+2)} Data in ASCII format INTEGER *4
The number of bytes actually transmitted. ( not counting the CR and LF, 
5. 3. 5 GPRDATSTR

Function:

Reads a character string containing data from the GPIB bus.

Calling Sequence:

GPIB_STATUS = GPRDATSTR ( GPIB_DEVICE_ADDR, DEVICE_CDUNT, 2 DATA_STRING, BYTES_TRANSMITTED，

Input/Output:

Input:

GPIB_DEVICE_ADDR

DEVICE_COUNT

Dutput:

DATA_STR ING

BYTES_TRANSMITTED
INTEGER*2 (DEVICE_COUNT)

Array of GPIB addresses

INTEGER* 4

The sum of the number of TALKERS and LISTENERS on the GPIB bus.

CHARACTER* (*) INTEGER $* 4$ data from GPIB

The number of bytes actualiy transmitted. ( not counting the CR and LF, 
5. 3. 6 GPTRIE

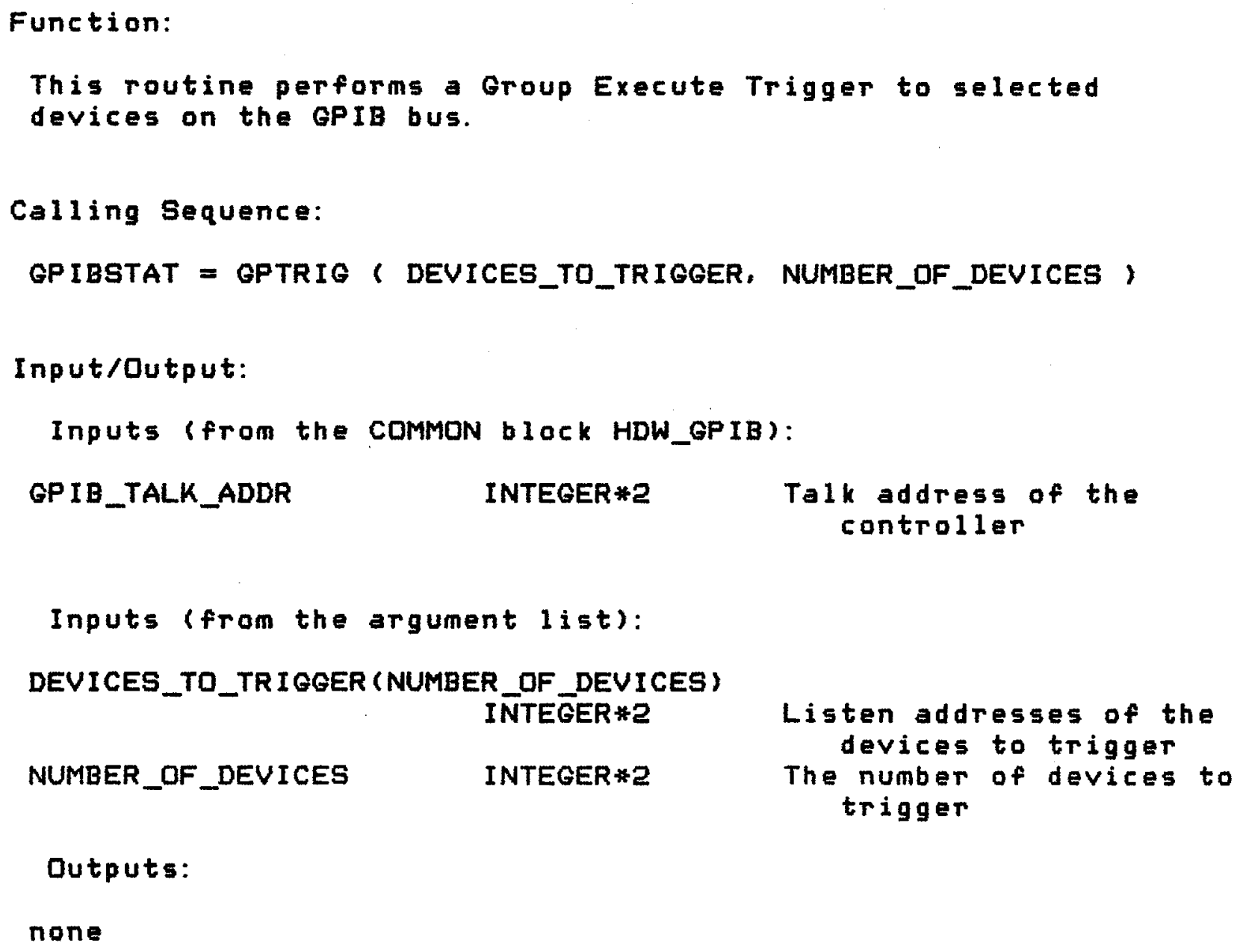

Dutputs:

none 


\section{3.7 GPULISTEN}

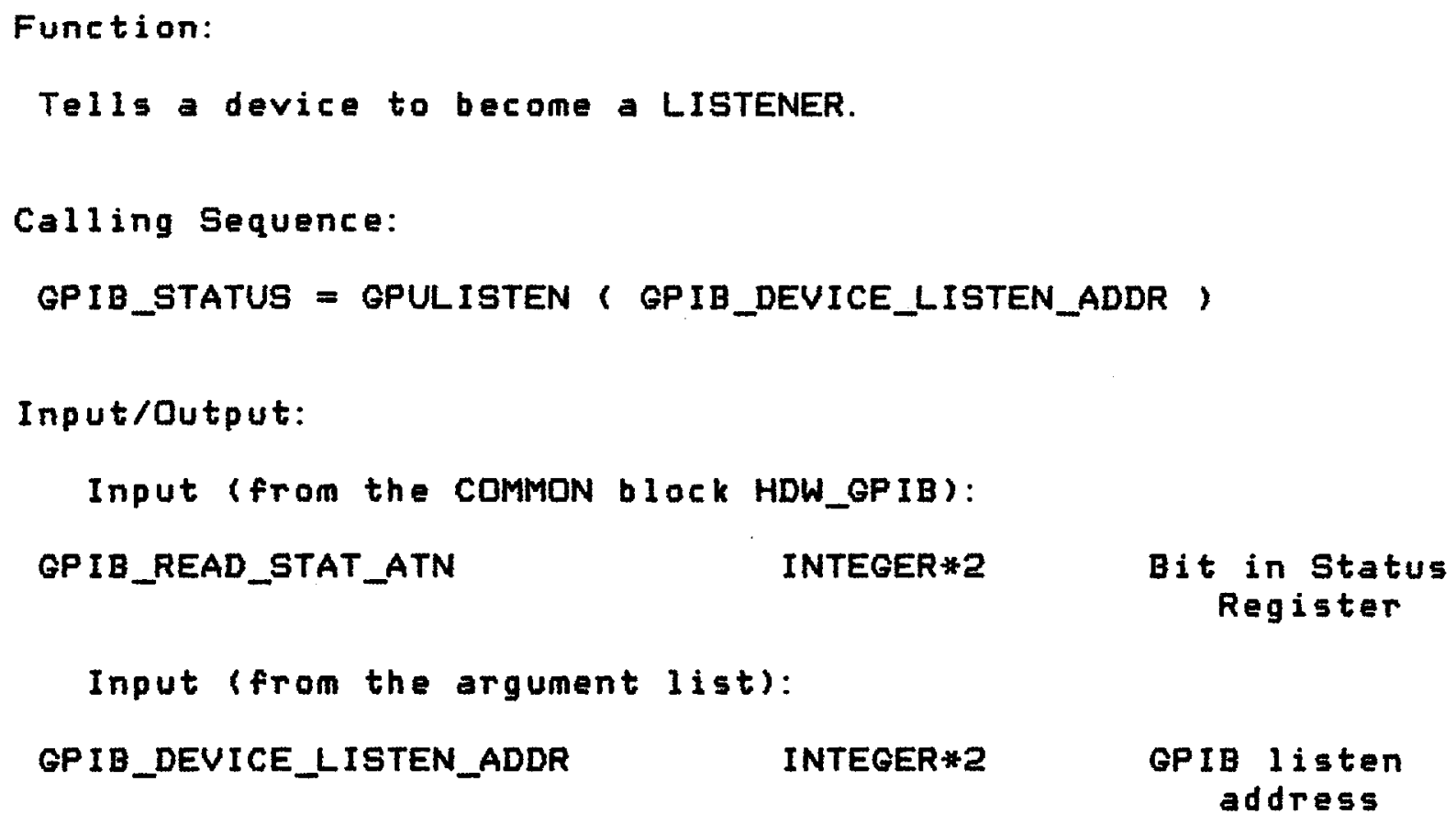


5. 3. 8 GPUNL

Function:

This routine UNLISTENs all devices on the GPIB bus.

Calling Sequence:

GPIBSTAT = GPUNL ( )

Input/Qutput:

Input (from the COMMON block HDW_GPIB):

GPIB_READ_STAT_ATN INTEGER*2

Bit in Status

Register

5. 3.9 GPUNT

Function:

This routine UNTALKs the current talker on the GPIB bus.

Calling Sequence:

GPIBSTAT = GPUNT ( )

Input/Output:

Input (from the COMMON block HDW_GPIB):

GPIB_READ_STAT_ATN INTEGER*2

Bit in Status

Register 
5. 3. 10 GPUTALK

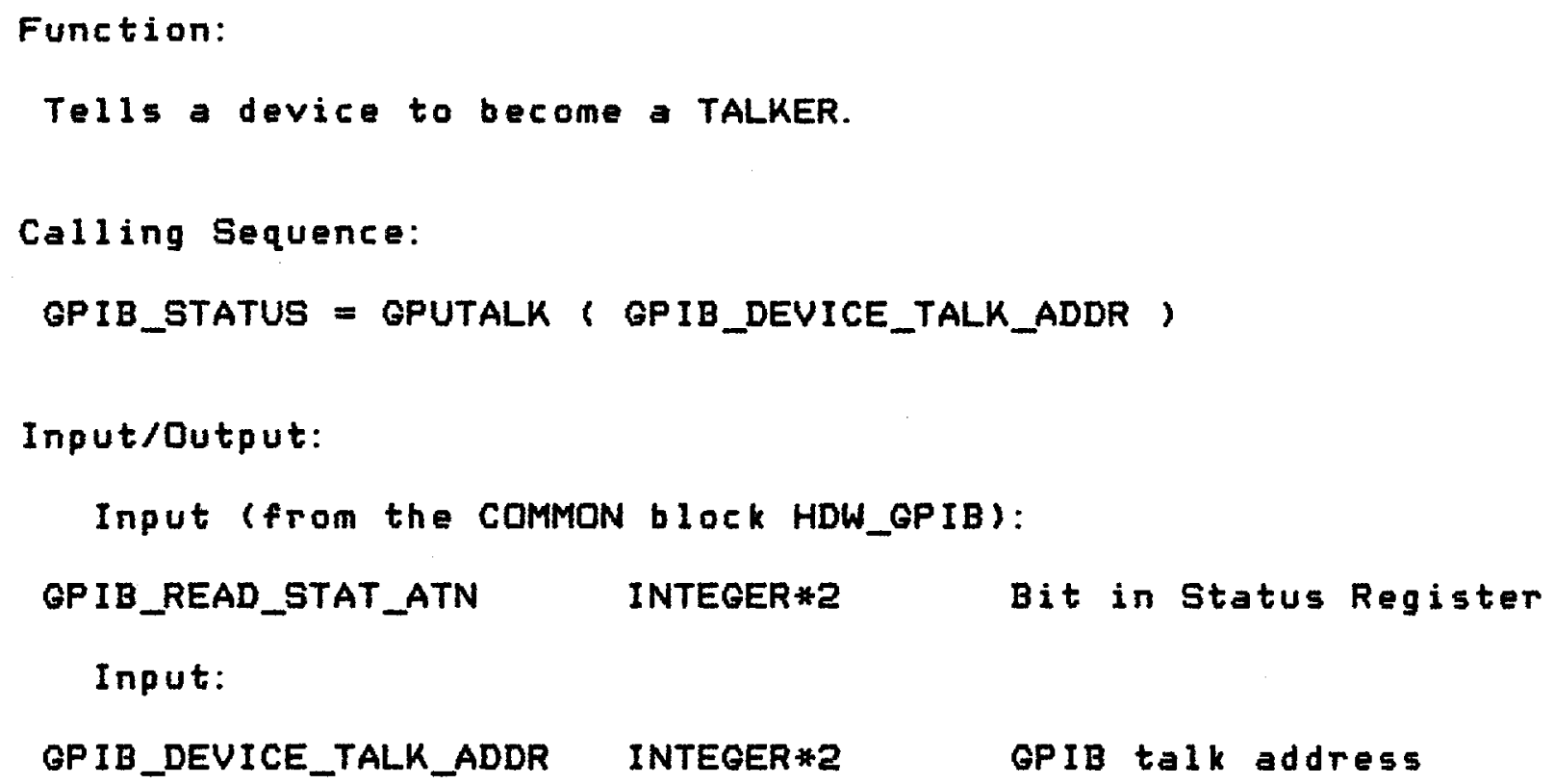




\section{3. 11 GPXMIT}

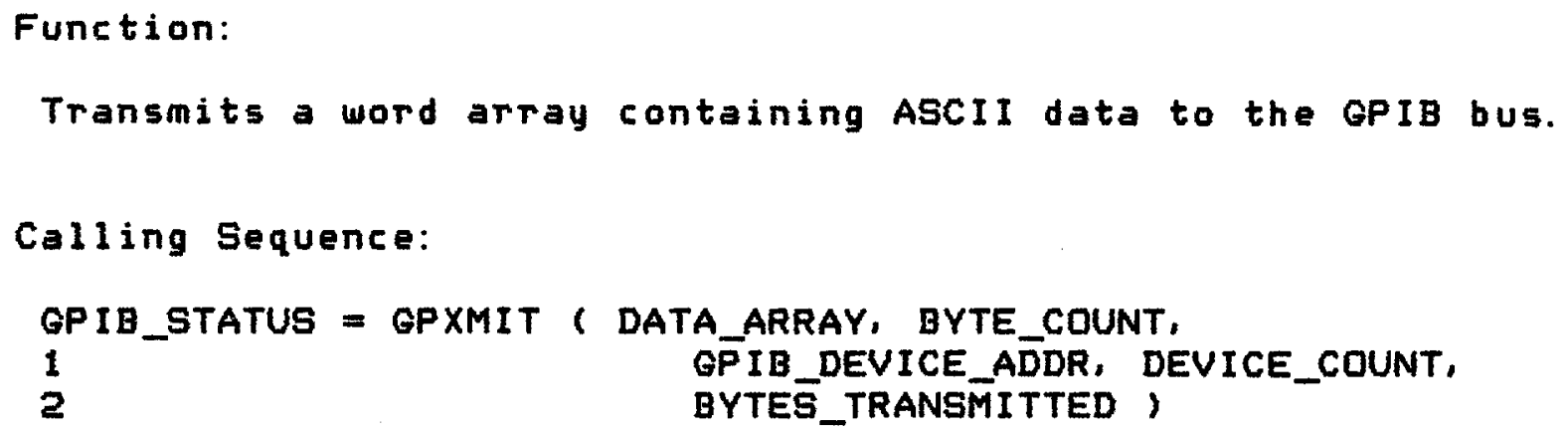

Input/Output:

Inputs (from the COMMON block HDW_GPIB):

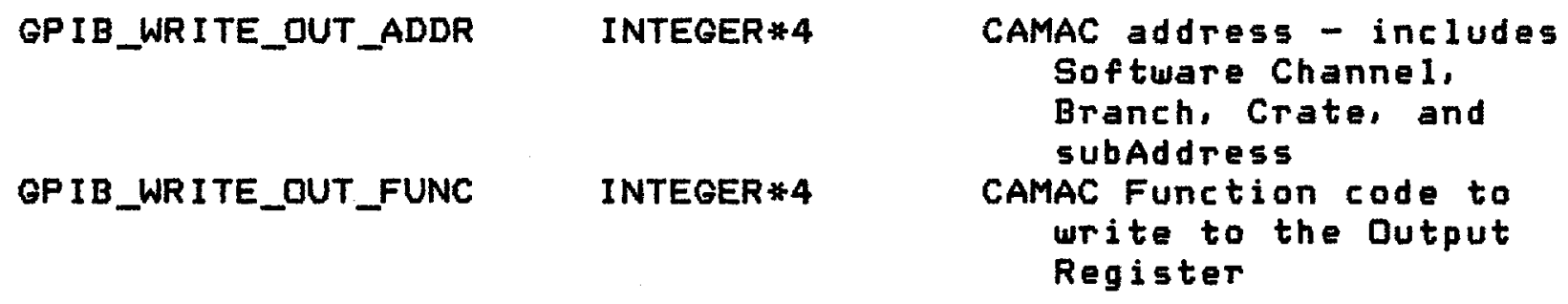

Inputs (from the argument list):

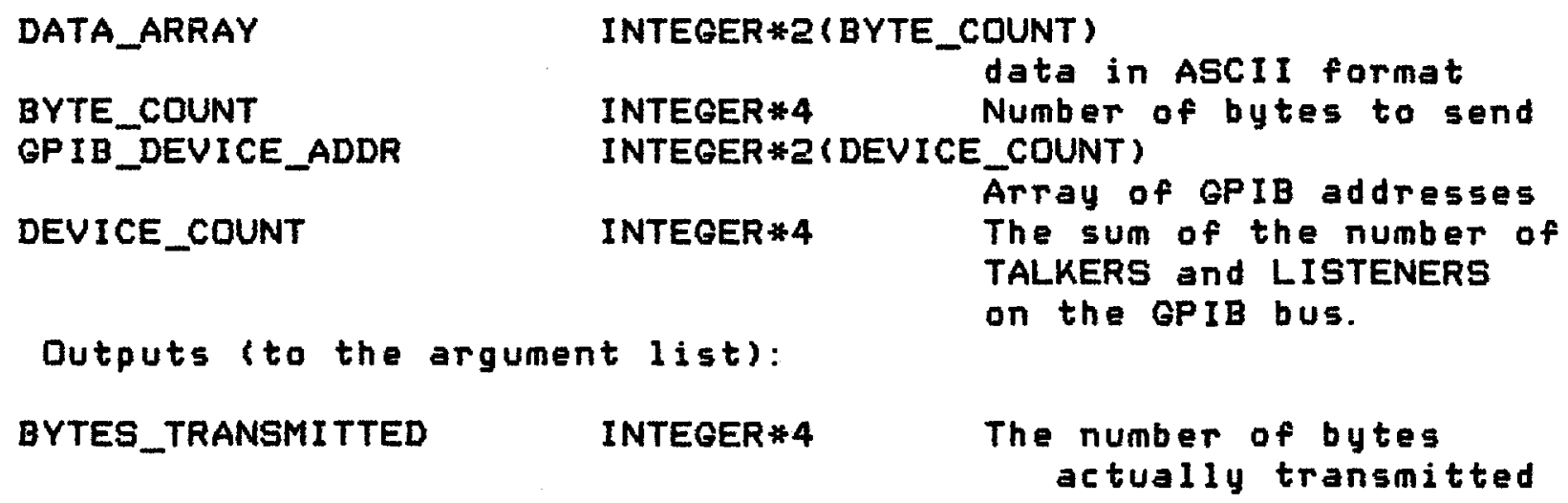




\section{3. 12 GPXMITSTR}

Function:

Transmits an ASCII string to the GPIB bus.

Calling Sequence:

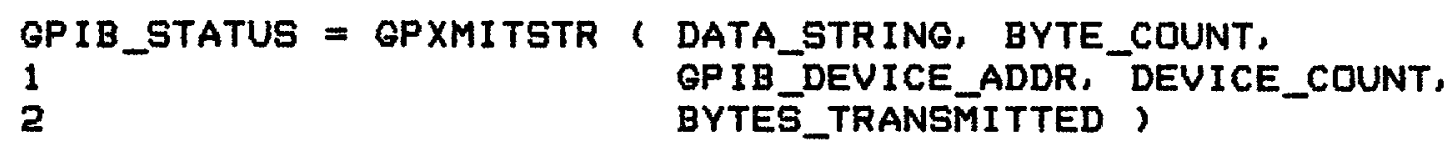

Input/Output:

Inputs (from the COMMON block HDW_GPIB):

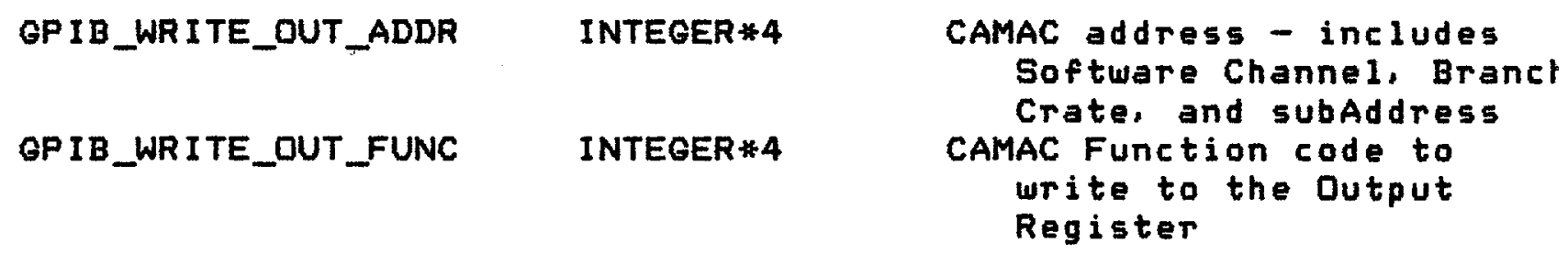

Inputs (from the argument list):

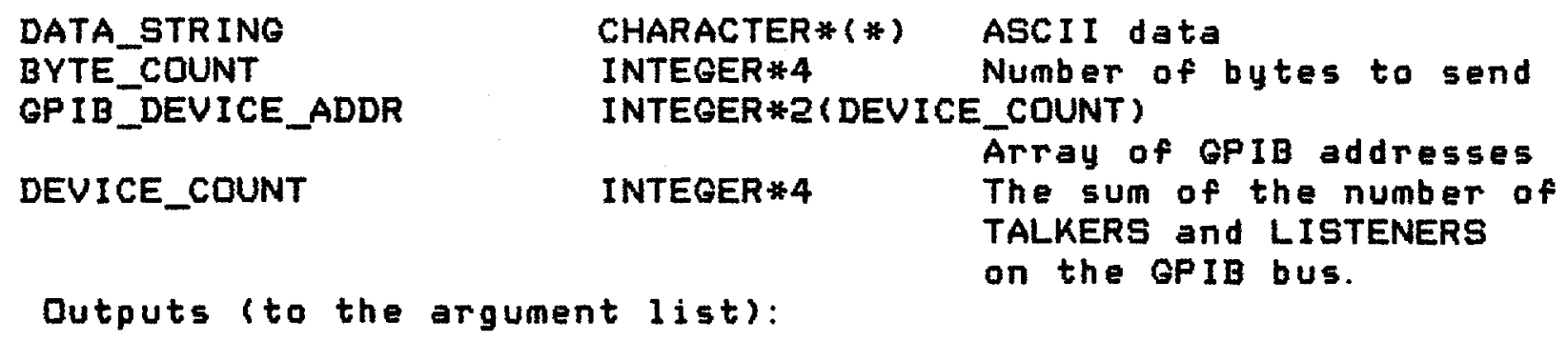

Dutputs (to the argument list):

BYTES_TRANSMITTED INTEGER*4

The number of bytes actually transmitted 